\title{
Safety and antibody response to two- dose SARS-CoV-2 messenger RNA vaccination in patients with multiple myeloma
}

Ross S. Greenberg', Jake A. Ruddy', Brian J. Boyarsky', William A. Werbel², Jacqueline M. Garonzik-Wang ${ }^{1}$, Dorry L. Segev ${ }^{1,3^{*}}$ and Philip H. Imus ${ }^{4}$

\begin{abstract}
Background: Patients with multiple myeloma (MM) were excluded from the original SARS-CoV-2 mRNA vaccine trials, which may influence vaccine hesitancy in this population. We prospectively characterized the safety and immunogenicity of two-dose SARS-CoV-2 mRNA vaccination in 44 patients with MM, who underwent vaccination from 12/17/2020 to 3/18/2021.
\end{abstract}

Results: Rates adverse reactions were low and consistent with those documented in vaccine trials. Among those on MM therapy, 93\% developed detectable anti-receptor binding domain (RBD) antibodies after dose 2, while 94\% of patients not on MM therapy seroconverted.

Conclusions: Two-dose SARS-CoV-2 mRNA vaccination is mildly reactogenic and leads to high rates of seroconversion in patients with MM. These findings can provide reassurance to MM patients who are hesitant to receive SARSCoV-2 mRNA vaccines.

Keywords: Multiple myeloma, COVID-19, SARS-CoV-2, mRNA vaccination, Antibody

\section{Introduction}

Patients with multiple myeloma (MM) have experienced high (34\%) inpatient mortality due to COVID-19 [1]. However, patients with $\mathrm{MM}$ and other immunocompromised populations were excluded from the SARSCoV-2 mRNA vaccine trials [2-4]. Lack of information about the safety and immunogenicity of the vaccines in patients with MM may contribute to vaccine hesitancy, and as such these data are critical to patients and their providers.

Reactogenicity of the SARS-CoV-2 mRNA vaccines in immunocompromised populations, such as solid organ transplant (SOT) and rheumatic and musculoskeletal

\footnotetext{
*Correspondence: dorry@jhmi.edu

${ }^{3}$ Department of Epidemiology, Johns Hopkins University Bloomberg

School of Public Health, Baltimore, MD, USA

Full list of author information is available at the end of the article
}

diseases (RMD) populations, appears similar to that of the immunocompetent people studied in the original vaccine trials [2-8]. However, the immunogenicity of the vaccines has been demonstrated to be decreased in these populations [5-8].

Patients with MM are on therapies that dampen the humoral and cellular immune responses, which has been linked to a diminished response to vaccines [9]. One recent study demonstrated $56 \%$ seroconversion at least 21 days following the first dose (D1) of the SARS-CoV-2 mRNA vaccine in the MM population, which is substantially lower than the $100 \%$ seroconversion observed in the original trials $[2-4,10]$. Two other groups found reduced post-D1 neutralizing antibody production in the elderly MM population, relative to healthy controls $(20.6 \% \mathrm{MM}$ vs. $32.5 \%$ control after 3 weeks; $78.6 \% \mathrm{MM}$ vs. $100 \%$ control after 5 weeks) $[11,12]$. In contrast to these three 
single-center reports on the mRNA vaccine in patients with $\mathrm{MM}$, the present study features a younger, national sample, as well as the safety and antibody response to two doses of mRNA vaccine [10-12]. We studied the safety and antibody response to two-dose SARS-CoV-2 mRNA vaccination in patients with MM.

\section{Materials and methods}

Patients who reported a diagnosis of $M M \geq 18$ years old without a history of COVID-19 were recruited to participate in this prospective cohort between 12/17/2020$3 / 18 / 2021$. Recruitment was conducted via a social media campaign. Diagnosis, demographics, and therapeutic regimens were collected via participant report and managed using the REDCap electronic data capture tool, a secure, web-based software platform designed to support data capture for research studies. One week after each dose, participants completed a questionnaire about local (pain, swelling, erythema) and systemic reactions (fatigue, headache, myalgia, chills, fever, diarrhea, vomiting) as well as adverse events (anaphylaxis, incident neurologic diagnoses, and infections including SARS-CoV-2).

One month after dose 2 (D2), participants underwent SARS-CoV-2 antibody testing via the Roche Elecsys $^{\circledR}$ anti-SARS-CoV-2S enzyme immunoassay which measures total antibody (IgM, IgG) to the SARSCoV-2S-receptor binding domain (RBD) protein, the target of the mRNA vaccines. The assay's detection limits ranged from $<0.4$ to $>250 \mathrm{U} / \mathrm{mL}$, with a positive result at $>0.79 \mathrm{U} / \mathrm{mL}$. This study was approved by the Johns Hopkins Institutional Review Board (IRB00248540) and participants provided informed consent electronically.

\section{Results}

We studied 44 patients with MM who received two-dose SARS-CoV-2 mRNA vaccination (Table 1 ). The median (IQR) age was 64 (57-69) years, 68\% were female, $98 \%$ were white, and $50 \%$ received the Pfizer/BioNTech vaccine while $50 \%$ received Moderna. The most common therapeutic regimens included lenalidomide (39\%), daratumumab (16\%), and pomalidomide (9\%), while $17(39 \%)$ were not on therapy.

The majority of local and systemic reactions were mild (80\% of all local reactions and $71 \%$ of all systemic reactions) (Supplemental Fig. 1). The most common local reaction was pain (75\% after D1 and $73 \%$ after D2). The most common systemic reactions were fatigue (39\% after D1 and 64\% after D2), headache (32\% after D1 and 50\% after D2), and myalgia (32\% after D1 and $41 \%$ after D2). Since vaccination, no participant developed anaphylaxis,

Table 1 Demographic and clinical characteristics of 44 patients with multiple myeloma, stratified by anti-SARS-CoV-2 RBD antibody response to two-dose SARS-CoV-2 mRNA vaccination

\begin{tabular}{|c|c|c|c|}
\hline & Overall $(n=44)$ & Detectable antibody $(n=41)$ & $\begin{array}{l}\text { Undetectable } \\
\text { antibody }{ }^{\mathrm{a}} \\
(n=3)\end{array}$ \\
\hline Age, median (IQR) & $64(57,69)$ & $64(57,69)$ & $58(55,58)$ \\
\hline Female, no. (\%) & $30(68)$ & $28(93)$ & $2(7)$ \\
\hline Non-white, no. (\%) & $1(2)$ & $1(100)$ & $0(0)$ \\
\hline \multicolumn{4}{|l|}{ Vaccine manufacturer, no. (\%) } \\
\hline Pfizer/BioNTech & $22(50)$ & $21(95)$ & $1(5)$ \\
\hline Moderna & $22(50)$ & $20(91)$ & $2(9)$ \\
\hline $\begin{array}{l}\text { Days from vaccine to antibody testing, median } \\
\text { (IQR) }\end{array}$ & $29(28,32)$ & $29(27,31)$ & $32(28,38)$ \\
\hline \multicolumn{4}{|l|}{ Therapy, no. (\%) } \\
\hline Not on therapy & $17(39)$ & $16(94)$ & $1(6)$ \\
\hline On therapy ${ }^{b}$ & $27(61)$ & $25(93)$ & $2(7)$ \\
\hline Bortezomib & $1(2)$ & $1(100)$ & $0(0)$ \\
\hline Carfilzomib & $1(2)$ & $1(100)$ & $0(0)$ \\
\hline Daratumumab & $7(16)$ & $7(100)$ & $0(0)$ \\
\hline Ixazomib & $2(5)$ & $1(50)$ & $1(50)$ \\
\hline Lenalidomide & $17(39)$ & $16(94)$ & $1(6)$ \\
\hline Pomalidomide & $4(9)$ & $4(100)$ & $0(0)$ \\
\hline Teclistamab & $1(2)$ & $0(0)$ & $1(100)$ \\
\hline
\end{tabular}

a The percentages in these columns are shown as percent of each category in the overall column. Detectable antibody is defined as an anti-SARS-CoV-2 RBD antibody titer $>0.79 \mathrm{U} / \mathrm{mL}$ by the manufacturer

b Since participants could report more than one therapy, the sum of the therapies is greater than the total $\mathrm{N}$ 
incident SARS-CoV-2, or a new neurologic condition or infection.

Forty-four participants underwent antibody testing at a median (IQR) of 29 (28-32) days after D2 (Table 1, Fig. 1, Supplemental Table 1). Anti-RBD was detectable in 41/44 (93\%; 95\% CI, 81-99\%) overall, 25/27 (93\%; 95\% CI, 76-99\%) of those on therapy, and 16/17 (94\%; 95\% CI, $71-99 \%)$ of those not on therapy. The median titer was above the upper limit of the assay $(>250 \mathrm{U} / \mathrm{mL})$ overall, for those on therapy, and for those not on therapy. The two participants on therapy that did not seroconvert were on teclistamab (a B-cell maturation antigen [BCMA]-CD3 bi-specific antibody in development) and lenalidomide/ixazomib.

\section{Discussion}

In this study of patients with MM, two-dose SARSCoV-2 mRNA vaccination resulted in expected systemic reactogenicity and high immunogenicity. Local and systemic adverse reactions were mostly mild ( $80 \%$ of local and $71 \%$ of systemic reactions), which is a trend consistent with what was reported in the vaccine clinical trials $[3,4]$. Local reactions were more common post-D1, and systemic reactions were more reported by more participants post-D2, which is also consistent with what was reported in the vaccine clinical trials $[3,4]$. We found high rates of seroconversion after two doses of the vaccine, and these did not vary based on whether patients were on MM therapy (93\% seroconversion on therapy vs. 94\% seroconversion without therapy). This contrasts with what was observed in another study post-D1, where $74 \%$ of patients without therapy seroconverted post-D1, while only $48 \%$ of those on therapy seroconverted [11].

While high immunogenicity in MM patients is promising, clinical trials in the general population reported a $100 \%$ seroconversion rate for both vaccine types $[3,4]$. Median antibody titers in immunocompetent populations in response to the vaccine measured by the same assay used in this study have been found to be $>250 \mathrm{U} /$ $\mathrm{mL}[13,14]$. Of note, the only participant on teclistamab did not develop an antibody response. Earlier studies found that lack of SARS-CoV-2 seroconversion occurs in patients on B-cell disruptive therapy [15]. Teclistamab induces $\mathrm{T}$ cell-mediated destruction of $\mathrm{B}$-cell maturation antigen (BCMA) positive B-cells [16]. Previous work has shown that lenalidomide can boost vaccine effectiveness in MM patients [17]. In this study, 94\% (16/17) of the patients on lenalidomide experienced positive seroconversion.

This study is limited by a small size, which limits the ability to compare titers between participants on different regimens. Furthermore, the assay's low dynamic range makes quantitative comparison of antibody titers difficult. Additionally, monoclonal protein concentrations were not measured, which have been correlated with vaccine response to other vaccines [18]. Due to

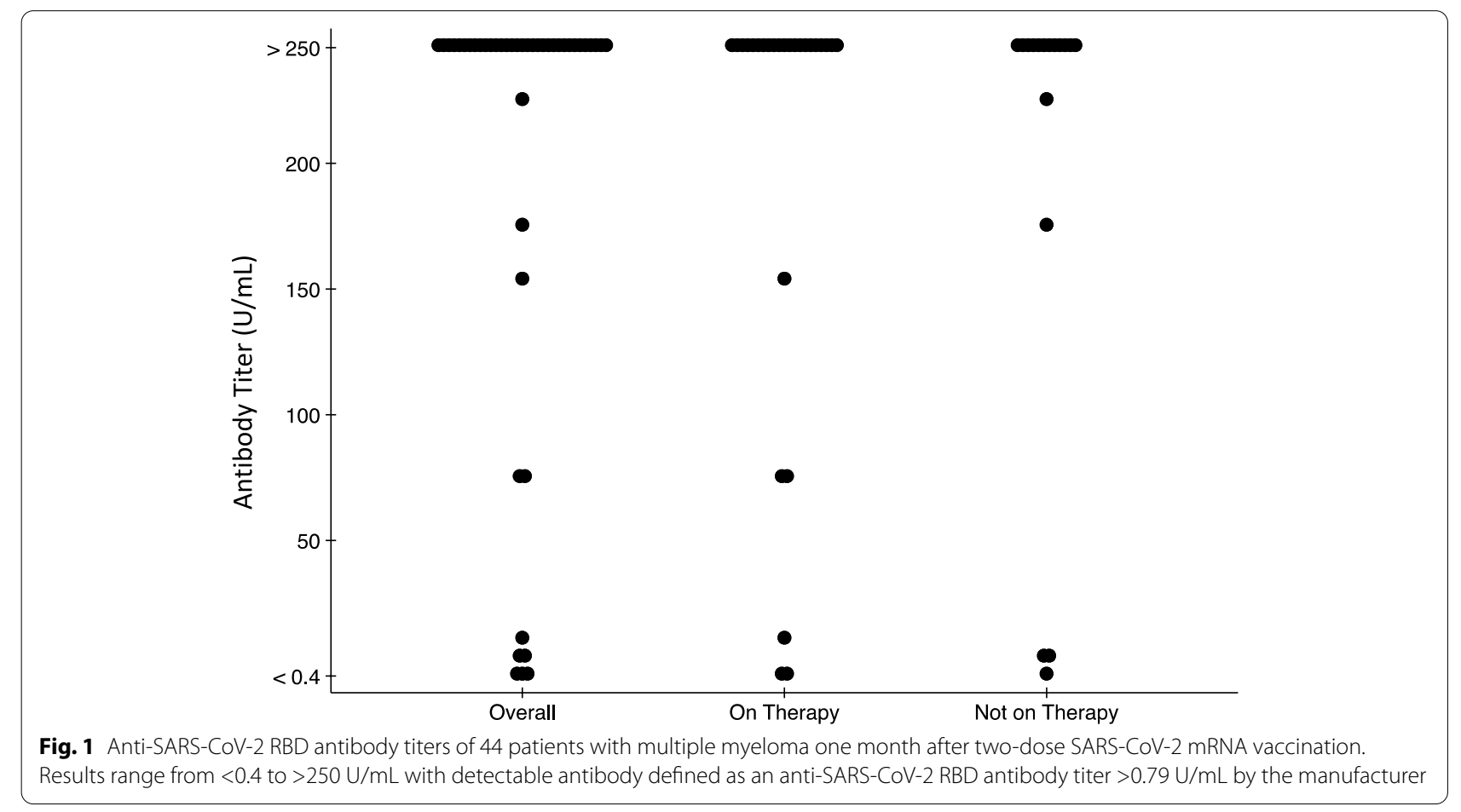


these limitations, we do not yet know the degree to which detectable antibody levels impact the clinical presentation and severity of COVID-19 in patients with MM. The present study presents evidence that vaccination against SARS-CoV-2 is safe for patients with MM. Additionally, increased anti-RBD antibody levels suggest that vaccination may decrease COVID-19 morbidity and mortality in this population. Social media recruitment and convenience sampling may have influenced the homogeneity of the study, as other papers from our group, with the same recruitment strategy, yielded similar population demographics [6-8]. Future investigations should include larger samples, examine the role of specific medications in vaccine response, and examine cellular immunity.

\section{Conclusion}

In conclusion, while patients with MM are at increased risk of COVID-19 disease burden and need to remain vigilant to protect themselves, this study provides evidence that vaccination with the BNT162b2 and mRNA1273 vaccines is safe and immunogenic in patients with MM. These results support the recommendation of SARS-CoV-2 mRNA vaccination for patients with MM.

Abbreviations

MM: Multiple myeloma; SOTR: Solid organ transplant recipients; D1: Dose 1; D2: Dose 2.

\section{Supplementary Information}

The online version contains supplementary material available at https://doi. org/10.1186/s12885-021-09097-5.

Additional file 1: Supplemental Figure 1. Local site and systemic adverse reactions in 44 patients with multiple myeloma within 7 days of dose 1 and dose 2 of the SARS-CoV-2 mRNA vaccine. Supplemental Table 1. Anti-SARS-CoV-2 RBD antibody titer of 44 patients multiple myeloma 1 month after two-dose SARS-CoV-2 mRNA vaccination by demographics and clinical characteristics.

\section{Acknowledgements}

We acknowledge the following individuals for their assistance with this study: Jennifer D. Motter MHS, Michael T. Ou BS, Aura T. Teles BS, and Michelle R. Krach MS.

\section{Authors' contributions}

R.S.G. and J.A.R. collected the data, J.A.R. analyzed the data, and all authors contributed to the planning, reporting, writing, and critical review of the work described in the article. The author(s) read and approved the final manuscript.

\section{Funding}

This research was made possible with generous support of the Ben-Dov family. This work was supported by grant number F32DK124941 (Boyarsky) and K23DK115908 (Garonzik-Wang) from the National Institute of Diabetes and Digestive and Kidney Diseases (NIDDK), and K24Al144954 (Segev) from National Institute of Allergy and Infectious Diseases (NIAID).

\section{Availability of data and materials}

The datasets used and/or analysed during the current study available from the corresponding author on reasonable request.

\section{Declarations}

Ethics approval and consent to participate

This study was approved by the Johns Hopkins Institutional Review Board (IRB00248540) and participants provided informed consent electronically. All methods were carried out in accordance with relevant guidelines and regulations.

\section{Consent for publication}

Not applicable.

\section{Competing interests}

D.L.S. has the following financial disclosures: consulting and speaking honoraria from Sanofi, Novartis, CSL Behring, Jazz Pharmaceuticals, Veloxis, Mallincrodt, Thermo Fisher Scientific. The other authors of this manuscript have no financial disclosures or conflicts of interest to disclose as described by BMC Cancer.

\section{Author details}

${ }^{1}$ Department of Surgery, Johns Hopkins University School of Medicine, Baltimore, MD, USA. ${ }^{2}$ Department of Infectious Diseases, Johns Hopkins University School of Medicine, Baltimore, MD, USA. ${ }^{3}$ Department of Epidemiology, Johns Hopkins University Bloomberg School of Public Health, Baltimore, MD, USA. ${ }^{4}$ Department of Oncology, Johns Hopkins University School of Medicine, Baltimore, MD, USA.

Received: 4 June 2021 Accepted: 2 December 2021

Published online: 27 December 2021
References

1. Martínez-López J, Mateos MV, Encinas C, Sureda A, Hernández-Rivas JÁ, de la Guía AL, et al. Multiple myeloma and SARS-CoV-2 infection: clinical characteristics and prognostic factors of inpatient mortality. Blood Cancer J. 2020;10(10):1-1.

2. Gavriatopoulou M, Ntanasis-Stathopoulos I, Korompoki E, Terpos E, Dimopoulos MA. SARS-CoV-2 Vaccines in Patients With Multiple Myeloma. HemaSphere 2021, 5(3):e547.

3. Polack FP, Thomas SJ, Kitchin N, Absalon J, Gurtman A, Lockhart S, et al. Safety and efficacy of the BNT162b2 mRNA Covid-19 vaccine. N Engl J Med. 2020;383(27):2603-15.

4. Walsh EE, Frenck RW, Falsey AR, Kitchin N, Absalon J, Gurtman A, et al. Safety and Immunogenicity of Two RNA-Based Covid-19 Vaccine Candidates. New England J Med. 2020;383(25):2439-50.

5. Connolly CM, Ruddy JA, Boyarsky BJ, Avery RK, Werbel WA, Segev DL, et al. Safety of the first dose of mRNA SARS-CoV-2 vaccines in patients with rheumatic and musculoskeletal diseases. Ann Rheum Dis. 2021;80(8):1100-1.

6. Ou MT, Boyarsky BJ, Motter JD, Greenberg RS, Teles AT, Ruddy JA, et al: Safety and Reactogenicity of 2 Doses of SARS-CoV-2 Vaccination in Solid Organ Transplant Recipients. Transplant. 2021;105(10):2170-4.

7. Boyarsky BJ, Werbel WA, Avery RK, Tobian AAR, Massie AB, Segev $D L$, et al. Immunogenicity of a Single Dose of SARS-CoV-2 Messenger RNA Vaccine in Solid Organ Transplant Recipients. Jama. 2021;325(17):1784-6.

8. Boyarsky BJ, Ruddy JA, Connolly CM, Ou MT, Werbel WA, GaronzikWang JM, et al. Antibody response to a single dose of SARS-CoV-2 mRNA vaccine in patients with rheumatic and musculoskeletal diseases. Ann Rheum Dis. 2021;80(8):1098-9.

9. Ludwig H, Boccadoro M, Moreau P, San-Miguel J, Cavo M, Pawlyn $C$, et al: Recommendations for vaccination in multiple myeloma: a consensus of the European Myeloma Network. Leukemia. 2021;35(1):31-44. 
10. Bird S, Panopoulou A, Shea RL, Tsui M, Saso R, Sud A, et al. Response to first vaccination against SARS-CoV-2 in patients with multiple myeloma. Lancet Haematol. 2021;8(6):e389-e392.

11. Terpos E, Trougakos IP, Gavriatopoulou M, Papassotiriou I, Sklirou AD, Ntanasis-Stathopoulos I, et al. Low neutralizing antibody responses against SARS-CoV-2 in older patients with myeloma after the first BNT162b2 vaccine dose. Blood. 2021;137(26):3674-6.

12. Pimpinelli F, Marchesi F, Piaggio G, Giannarelli D, Papa E, Falcucci P, et al. Fifth-week immunogenicity and safety of anti-SARS-CoV-2 BNT162b2 vaccine in patients with multiple myeloma and myeloproliferative malignancies on active treatment: preliminary data from a single institution. J Hematol Oncol. 2021;14(1):1-2.

13. Mueller T. Antibodies against severe acute respiratory syndrome coronavirus type 2 (SARS-CoV-2) in individuals with and without COVID-19 vaccination: a method comparison of two different commercially available serological assays from the same manufacturer. Clin Chim Acta. 2021;518:9-16.

14. Cavalcanti E, Isgrò MA, Rea D, et al. Vaccination strategy and anti - SARSCoV-2S titers in healthcare workers of the INT - IRCCS "Fondazione Pascale" Cancer center (Naples, Italy). Infect Agent Cancer. 2021;16(1):32 Published 2021 May 12.

15. Deepak P, Kim W, Paley MA, Yang M, Carvidi AB, Demissie EG, et al. Effect of immunosuppression on the immunogenicity of mRNA vaccines to SARS-CoV-2: a prospective cohort study. Ann Int Med. 2021;174(11):1572-85.

16. Pillarisetti $K$, Powers $G$, Luistro L, Babich A, Baldwin E, Li Y, et al. Teclistamab is an active $T$ cell-redirecting bispecific antibody against B-cell maturation antigen for multiple myeloma. Blood Adv. 2020;4(18):4538-49.

17. Noonan K, Rudraraju L, Ferguson A, Emerling A, Pasetti MF, Huff CA, et al. Lenalidomide-induced immunomodulation in multiple myeloma: impact on vaccines and antitumor responses. Clin Cancer Res. 2012;18(5):1426-34.

18. Tete SM, Kipling D, Westra J, et al. Monoclonal paraprotein influences baseline B-cell repertoire diversity and perturbates influenza vaccinationinduced B-cell response. Exp Hematol. 2015;43(6):439-47.e1.

\section{Publisher's Note}

Springer Nature remains neutral with regard to jurisdictional claims in published maps and institutional affiliations.

Ready to submit your research? Choose BMC and benefit from:

- fast, convenient online submission

- thorough peer review by experienced researchers in your field

- rapid publication on acceptance

- support for research data, including large and complex data types

- gold Open Access which fosters wider collaboration and increased citations

- maximum visibility for your research: over $100 \mathrm{M}$ website views per year

At BMC, research is always in progress.

Learn more biomedcentral.com/submissions 